\title{
Clinical efficacy of the brachial plexus via posterior approach for orthopedic surgery
}

\section{Introduction}

The brachial plexus can be performed for various upper limb surgeries and depending on the surgical site; various approaches can be used as the interscalenic, supraclavicular or axillary pathway. ${ }^{1}$ The approach can be used with neurostimulator or ultrasound. Posterior brachial plexus block was described in $1912^{2}$ and reused in $1990^{3}$ by the loss of resistance technique and in 2005 as a continuous technique and neurostimulator. ${ }^{4}$

In 2005, the posterior approach of the brachial plexus was used in Brazil with neurostimulator and ropivacaine, providing effective anesthesia that is comfortable for the patient without the need for general anesthesia. ${ }^{5}$ In another study with the later approach with nerve-stimulation using different volumes of ropivacaine ${ }^{6}$ or same volume at different concentrations of ropivacaine ${ }^{7}$ both promoted similar postoperative analgesia. Although the posterior approach is deeper than the classic interesalenic approach, the use of the posterior approach can be easily identified by anatomical marking, which allows for complications of the anterior approach. The discomfort during the procedure to provide peripheral block occurs even after preanesthetic evaluation and the use of a comfortable medication. It has recently been shown that dextroketamine in subdoses provide sufficient sedation to maintain the patient in position for posterior brachial plexus block and for the relief of pain in 55\% of them during the procedure, without hemodynamic variation. ${ }^{8}$

This prospective and observational study was performed to evaluate the clinical effectiveness of posterior brachial plexus access with a single injection of the combination of lidocaine and enantiomeric excess levobupivacaine with neurostimulator in patients undergoing clavicle, shoulder and proximal chair procedures of beach associated with general anesthesia.

\section{Methods}

The study was submitted and approved by the Ethics Research Committee (Number 05787/2014) and all patients were explained the method and obtained their consent by signing the informed consent form. The study was conducted between January 2015 to December 2018 with 50 patients of physical status I and II (ASA) of both sexes who underwent elective clavicle, shoulder and proximal humeral procedures in beach chairs associated with general anesthesia. The protocol was registered in the Brazil Platform (CAAE: 33373214.1.0000.5179). As part of Program ACERTO, all patients drank a single $200 \mathrm{~mL}$ liquid oral hypercaloric nutritional supplement $(1.5 \mathrm{Kcal} / \mathrm{mL})$ without residue, clarified and without addition of lipid and fiber (Fresubin Jucy ${ }^{\circledR}$ ) about 2 to 4 hours before surgery. The sample size was based on the conservative calculation the population size of $95 \%$ and with a margin of error of $10 \%$, obtaining the number of 50 patients, ages 18 to 60 years, weighing 60 to $80 \mathrm{~kg}, 160$ to 180 $\mathrm{cm}$ tall.

Exclusion criteria for posterior brachial plexus block were the presence of allergy to local anesthetics, bronchopulmonary disease, coagulopathy or neurological diseases with brachial plexus damage and emergency or classified surgery patients as class III or more
Volume I 2 Issue I - 2020

\author{
Luiz Eduardo Imbelloni,' Douglas M. P. \\ Teixeira, ${ }^{2}$ Umberto Lima, ${ }^{2}$ Marcos Guedes \\ Miranda Junior, ${ }^{3}$ Ana Raquel Araruna Soares, ${ }^{3}$ \\ Geraldo Borges de Morais Filho ${ }^{4}$ \\ 'Anesthesiologist of Hospital Clínicas Municipal José de Alencar, \\ Brazil \\ ${ }^{2}$ Orthopaedic Surgeon of Complexo Hospitalar Mangabeira \\ Gov. Tarcisio Burity, Brazil \\ ${ }^{3}$ Resident in Anesthesiology of Hospital Clínicas Municipal José \\ de Alencar, Brazil \\ ${ }^{4}$ Master in Labour Economics, UFPB, Statistician of the \\ Complexo Hospitalar Mangabeira, Brazil
}

Correspondence: Dr. Luiz Eduardo Imbelloni, Anesthesiologist of Hospital Clínicas Municipal José de Alencar, Rua dos Coroados, 162 - Apto 45 - Bloco B,Vila Anastácio (Lapa), São Bernardo do Campo, 05092-020 - São Paulo, SP, Brazil,Tel + 55.83.99962-4I0I,Email dr.imbeloni@hotmail.com

Received: December 27, 2019 | Published: February 03, 2020

of ASA physical state. All the blocks were performed using the landmarks as Figure 1.

No patient received pre-anesthetic medication in the ward. Upon arrival in the operating room all patients were monitored with NIBP, electrocardiography, and pulse oximetry. After monitoring, an intravenous cannula ( $18 \mathrm{G}$ or $20 \mathrm{G})$ was placed in the contralateral upper limb and lactated Ringer's solution was started for volume replacement. All patients were initially sedated with intravenous midazolam $1 \mathrm{mg}$ and before placement in the puncture position for blockade with $0.1 \mu \mathrm{g} / \mathrm{kg}$ dextroketamine. ${ }^{8}$ Immediately after placement in the sitting position the puncture site was identified and marked. With aseptic condition, the plexus block was performed with a needle A100 needle gauge $21 \mathrm{G}$ with a cutting bevel of $30^{\circ}$ with 1 $\mathrm{mL}$ of $1 \%$ lidocaine infiltration, coupled to a NE. After performing the lidocaine needle entry path, the needle was introduced longitudinally between the sixth and seventh cervical vertebra and $3 \mathrm{~cm}$ off the midline (Figure 1).

The needle was advanced until a stimulus of the suprascapular nerve was excited with a stimulus of $0.6 \mathrm{~mA}$ and frequency was $2 \mathrm{~Hz}$, duration of the stimulating pulse was 0.3 milliseconds and a response observed. After a negative aspiration for blood, $20 \mathrm{~mL}$ of lidocaine $2 \%$ plus epinephrine (Cristália Produtos Químicos e Farmacêuticos Ltda) and $20 \mathrm{~mL}$ of enantiomeric excess levobupivacaine (S75: R25) $0.5 \%$ (Cristália Produtos Químicos e Farmacêuticos Ltda) were injected. The attempt to access the needle to obtain the desired stimulus was evaluated in number of corrective maneuvers until the desired motor response was obtained. And it was measured with a $10 \mathrm{~cm}$ ruler the distance obtained by the needle until obtaining the stimulus with NE.

The distribution of blockage in each dermatome of each nerve (Figure 2) was performed by a separate investigator at 20 minutes 
and the end of surgery. Similarly the upper limb block motor was evaluated by modified Bromage's scale: $0=$ no block, total arm and forearm flexion; $1=$ partial block, total forearm and partial arm flexion;
$2=$ almost complete block, inability to flex the arm and decreased ability to flex the forearm; $3=$ total block, inability to flex both arm and forearm at 20 minutes and at surgery completion.

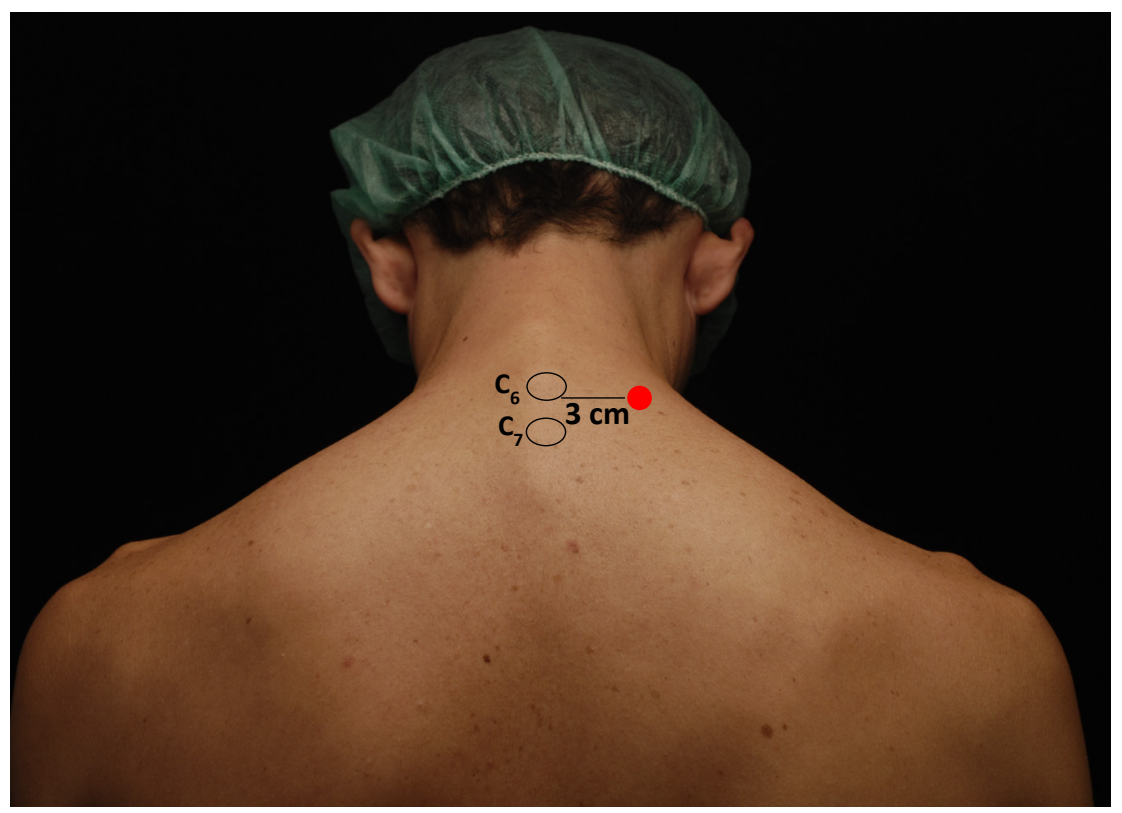

Figure I Anatomic landmarks for the posterior approach of the interscalene block. C6-C7, spinous processes of the sixth and seventh cervical vertebrae.

Site of puncture (needle/entry site)

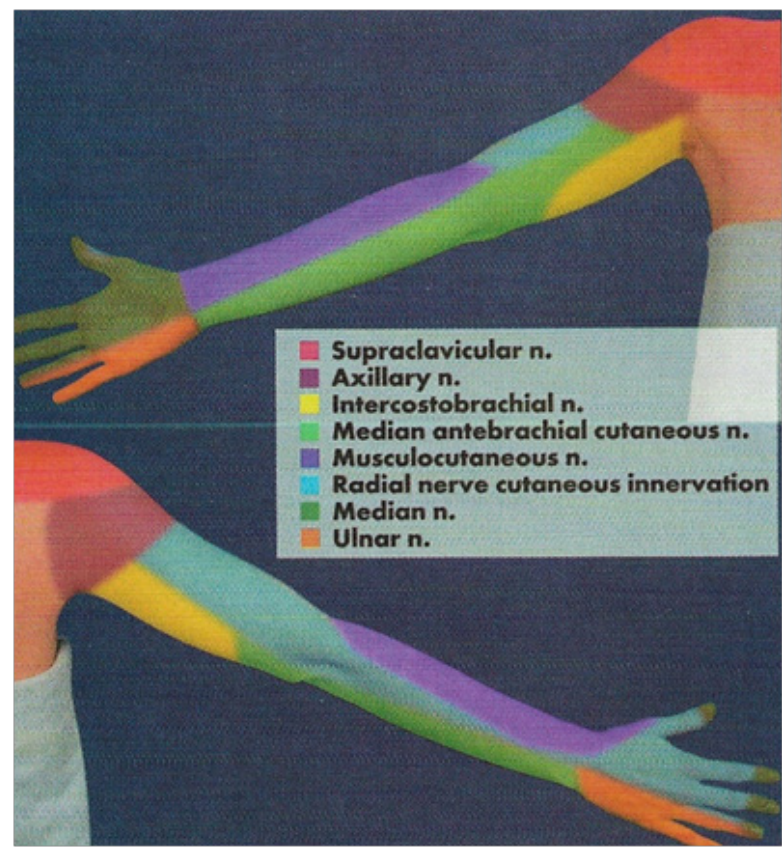

Figure 2 Peripheral nerve innervation (anterior and posterior surfaces).

The following parameters were also evaluated: 1) onset anesthesia, considered the time between anesthetic injection (lidocaine and levobupivacaine) and loss of sensitivity in the studied area of the axillary nerve; 2) surgery duration was considered the time between anesthetic injection and end of the surgical procedure and extubation; 3) sensory block duration, considered the time between anesthetic injection and total sensitivity recovery evaluated by painful stimulation in the operated limb; 4) motor block duration, considered as the time between the beginning of the loss of sensitivity and total recovery of movements; 5) duration analgesia, considered as the time between onset and the first analgesic request by patient; 6) complications and side effects. Posterior brachial block failure was defined as absence of anesthesia in areas supplied by supraclavicular, suprascapular and axillary nerves 20 minutes after local anesthetic injection, when general anesthesia was induced.

All surgical procedures were performed in the beach chair position and according to protocol after evaluation of anesthetized dermatomes at 20 minutes, all patients underwent general balanced anesthesia with propofol $(2.0 \mathrm{mg} / \mathrm{kg})$, fentanyl $(20 \mu \mathrm{g} / \mathrm{kg}))$, and cisatracurium $(0.15 \mathrm{mg} / \mathrm{kg})$ and sevoflurane was used for anesthesia maintenance. Ondansetron, $4 \mathrm{mg}$ and dexamethasone, $10 \mathrm{mg}$, and were used for the prophylaxis of nausea and vomiting. At the end of the surgery, patients were transferred to the PACU and after blockade recovery was referred to the ward. In case of pain; intravenous ketoprofen (100 $\mathrm{mg}$ ) and dypirone (2 g) were administered. Patients were followed for 48 hours to evaluate complications at site blockade and satisfaction with the technique. All patients were followed by telephone for 30 days. In the present study the statistical analysis was performed with the nonparametric Wilcoxon test with Spearman correlation and the McNemar with Yates correction test, being considered significant the value of $\mathrm{p}<0.05$. 


\section{Results}

Sixty-seven patients participated in the study and after using the exclusion criteria 50 patients were included in the final study. Patient recruitment and flow are summarized in Figure 3. The demographics data are shown in Table 1. The latency time (onset of analgesia) was 3:50 minutes and the surgical procedure was 2:20 hour. Mean analgesia duration was 18 hours. During the surgical procedure no failure was observed during general anesthesia in any patient, and no patient required fentanyl supplementation (Table 2). The success in posterior brachial plexus localization with $\mathrm{NE}$ at the first attempt occurred in $40(80 \%)$ of the patients. Subsequently two attempts were required in $5(10 \%)$ patients, three attempts in $3(6 \%)$ patients, and finally four attempts in $2(4 \%)$ patients, respectively. In all patients the first stimulus was always suprascapular with intensity of $0.6 \mathrm{~mA}$. Neck pain during needle entry was not observed in any patient.

\section{ENROLLEMENT}

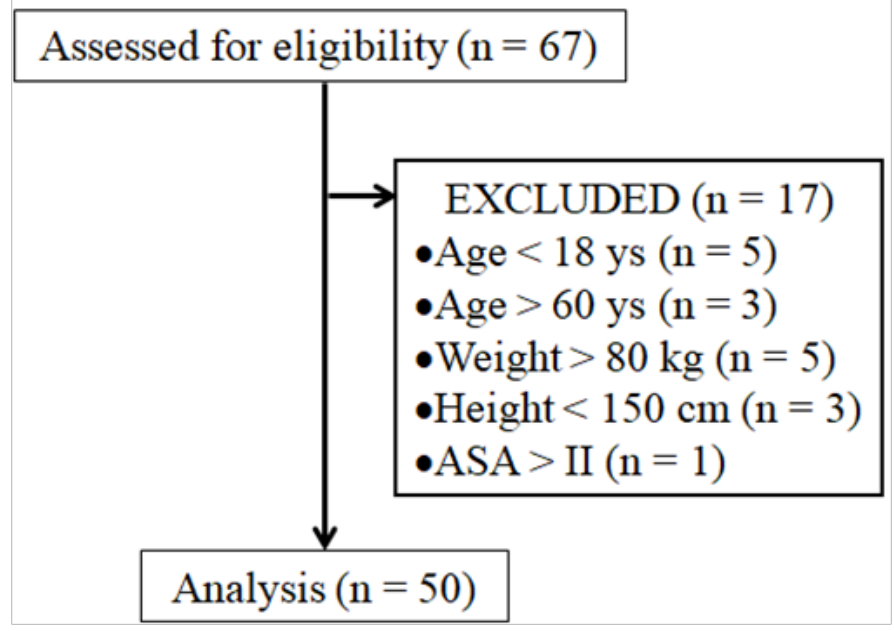

Figure 3 Consolidated Standards of Reporting Trials (CONSORT) flow diagram.

Table I Demographics Data

\begin{tabular}{ll}
\hline Variables & Results \\
\hline Age (years) & $37.18 \pm 13.11$ \\
Weight $(\mathrm{kg})$ & $66.68 \pm 9.18$ \\
Height $(\mathrm{cm})$ & $166.24 \pm 9.01$ \\
Gender: Male/Female & $33 / 17$ \\
Physical Status:ASA (I/I) & $21 / 29$ \\
\hline
\end{tabular}

Blockade in each dermatome evaluated at 20 minutes and at the end of the surgical procedure are in Table III and only the supraclavicular and axillary nerves were blocked in all patients, respectively. Statistical analysis showed a significant increase in the number of dermatomes blocked when compared to 20 minutes with the end of surgery. This increase was not observed with the musculocutaneous nerve. Anesthesia was adequate in all 50 patients, and no failure occurred during surgery without the need for opioid supplementation.
Motor block by Bromage modified range for upper limb at 20 minutes was absent (level 0) in all patients, level 1 in $25(50 \%)$ out of 50 patients, level 2 in $21(42 \%)$ out of 50 patients and level 3 in $4(8 \%)$ out of 50 patients. The motor block assessed at the end of surgery showed a different outcome compared to 20 minutes and there was a motor block level 0 and level 1 in 0 out of 50 patients, level 2 in 17 (34\%) out of 50 patients and level 3 in $33(66 \%)$ out of 50 patients (Table 3). There is a significant correlation between increased motor block at 20 minutes compared with the end of surgery (Wilcoxon test with Spearman correlation).

Table 2 Onset Anesthesia, Surgical and Analgesic Length Failure Block

\begin{tabular}{ll}
\hline Variables & Results \\
\hline Onset Anesthesia (min) & $3: 50 \pm 0: 45$ \\
Surgical Duration (hours) & $2: 20 \pm 0: 37$ \\
Analgesia Duration (hours) & $18 \pm 4$ \\
Failure Block & No \\
\hline
\end{tabular}

Table 3 Effects of Posterior Brachial Block

\begin{tabular}{|c|c|c|c|}
\hline Variables & 20 minutes & End Surgery & P-Value \\
\hline \multicolumn{4}{|l|}{ Sensory Block } \\
\hline Supraclavicular n. & $50(100 \%)$ & $50(100 \%)$ & \\
\hline Axillary n. & $50(100 \%)$ & $50(100 \%)$ & - \\
\hline Intercostobrachial n. & 35 (70\%) & 37 (74\%) & - \\
\hline $\begin{array}{l}\text { Median antebrachial } \\
\text { cutaneous } \mathrm{n} \text {. }\end{array}$ & 14 (28\%) & $36(72 \%)$ & $\begin{array}{l}0.5789 * \\
0.00001814 *\end{array}$ \\
\hline Musculocutaneous $\mathrm{n}$. & 35 (70\%) & 42 (84\%) & $0.1213^{*}$ \\
\hline Radial n. & 35 (70\%) & 46 (92\%) & $0.009823 *$ \\
\hline Median n. & $20(40 \%)$ & 43 (86\%) & $0.00007772 *$ \\
\hline Ulnar $\mathrm{n}$. & $18(36 \%)$ & $35(70 \%)$ & \\
\hline \multicolumn{4}{|l|}{ Motor Block } \\
\hline MB 0 & $0(0 \%)$ & $0(0 \%)$ & \\
\hline MB I & $25(50 \%)$ & $0(0 \%)$ & \\
\hline MB 2 & $21(42 \%)$ & 17 (34\%) & $0.0000 * *$ \\
\hline MB 3 & $4(8 \%)$ & $33(66 \%)$ & \\
\hline
\end{tabular}

* McNemar test with Yates correction

**Wilcoxon test

At 20 minutes only one patient presented Horner's syndrome and dysphonia. None patient experienced difficulty with breathing. At this time all patients received general anesthesia. After the end of the surgical procedure and after extubation in the patient had Horner's syndrome or respiratory distress. All patients were followed for 30 days follow-up and no local or systemic complications were observed. Regarding the technique used, all patients were satisfied. During the surgical procedure no patient presented side effects such as hypotension, arrhythmias or other signs and symptoms of accidental 
intravascular injection, such as dizziness, tinnitus, perioral tingling, metal taste, irritability, shivering or seizure. No patient had epidural or subarachnoid injection of local anesthetic.

\section{Discussion}

Since the first reports of brachial plexus block, it has been indispensable for regional anesthesia techniques used for upper limb procedures. Several ways of approaching the brachial plexus for proportional surgical procedures are suitable for postoperative anesthesia and analgesia, being widely performed for orthopedic procedures and outpatient surgery. In a recent article, the author shows that paravertebral cervical block differs from the interscalenic approach, since the posterior roots and not the anterior ones are better anesthetized. ${ }^{9}$ In a randomized controlled trial aiming at comparing the two approaches of the brachial plexus, no differences were found regarding block success, extent of the block, as well as onset and offset times..$^{10}$ In this study, using brachial plexus posterior access block with neurostimulator and the mixture of $2 \%$ lidocaine with $0.5 \%$ levobupivacaine (S75: R25), 100\% adequate anesthesia was obtained, and no blockade failure occurring. Posterior brachial plexus block with NE has promoted effective anesthesia for clavicle, shoulder, and proximal humerus procedures. Patients were comfortably operated in the seating position associated with general anesthesia.

Posterior brachial plexus block can be performed in both lateral decubitus ${ }^{5}$ and sitting position. ${ }^{2}$ In this study, the sitting position was used, being placed in this position after sedation with midazolam and dextroketamine. To facilitate the palpation of the spinous processes of $\mathrm{C} 6$ and $\mathrm{C} 7$ and marking the place to be punctured the neck was flexed. With a millimeter rule was marked three centimeters lateral at the bottom of $\mathrm{C} 6$ being the paravertebral line is traced in a cephalocaudal axis. This often corresponds to the groove between the levator scapulae and trapezius muscles. The puncture site is located on the midpoint of this paravertebral line. Our study showed that $100 \%$ of the patients had motor response of the supraclavicular nerve, resulting in complete motor of the supraclavicular and axillary nerves at 20 minutes and at the end of surgery. This fact can be justified by the blockade at the level of the sixth cervical roots, which becomes the middle trunk and posterior cord of the brachial plexus. The use of only one stimulation obtained with neurostimulation technique resulted in a rapid onset of block, complete block of the axillary, radial, median, and musculocutaneous nerves in our study, suggesting that a multistimulation technique associated with the later approach would not be justified nor necessary. There was a significant difference between the first 20 minutes and the end of surgery in the percentage and quality of blockade in the various nerves. This data confirms that the time for complete block installation is longer than 20 minutes. Our results at 20 minutes and at the end of surgery confirm that from other authors $^{7}$ who obtained $100 \%$ only from suprascapular and axillary nerve blocks.

The pain reported at the needle path site reported in the initial study $^{2}$ seems to have been a reason why this access route to the brachial plexus has not gained popularity, despite the fact that it is easy to perform, with a high incidence of success and with few complications. Another article using the original marking for posterior brachial plexus reported incidence of needle path pain for continuous blockade. ${ }^{4}$ One of the major disadvantages of brachial plexus block is the need to reach the first stimulus, which ranges from 4 to 7 centimeters, requiring the needle to pass through various muscles and may cause pain. Otherwise, the needle needs to pass through the scalene muscle causing injury to the long and dorsal scapular thoracic nerve. In our study, we used local infiltration with lidocaine and dextroketamine at a dose of $0.1 \mathrm{mg} / \mathrm{kg}^{8}$ for subsequent blockade needle entry (A100), and no patient reported postoperative neck pain, unlike $3 \%$ in another article. ${ }^{11}$ Our group has long experience with the technique using nerve stimulation with several published articles. ${ }^{5,8}$ This fact was confirmed in this series of 50 patients with $80 \%$ of the first attempts to be successful in locating the brachial plexus, similar results and those of other authors. ${ }^{11}$ In another article with catheter insertion through the needle, neck pain was caused by penetrating the extensor paraspinal muscles of the neck. ${ }^{4}$ This fact was not observed in this study with the single-injection nervestimulation technique used after lidocaine pathway infiltration and dextroketamine sedation.

In 2005 , studying 120 patients using the technique described above $^{11}$ for catheter use, showed that to provide good analgesia with a peak mean visual analogue scale pain score in the first $48 \mathrm{~h}$ of $17 \mathrm{~mm}$, and with a low incidence of side effects. Because of the proximity of the sympathetic cervical chain and the recurrent laryngeal nerve, Horner's syndrome and hoarseness can occur. In this study of 120 patients, only four had Horner's syndrome and one experienced difficulty breathing, and three patients described neck pain, which disappeared once the catheter was removed. In our single puncture study, we observed a case of Horner 'syndrome, without difficulty breathing for 20 minutes, and no neck pain.

The administration of the $20 \mathrm{~mL}$ association of $1 \%$ lidocaine with $20 \mathrm{~mL}$ of $0.25 \%$ levobupivacaine (R25: S75) provided a rapid onset of action (3.5 minutes) with mean duration of 18-hour analgesia, varying from 12 to 26 hours, without need for opioids, being considered adequate and long-lasting, allowing for early hospital discharge in less than 24 hours for some patients. The administration of the same volume $(40 \mathrm{~mL})$ as $0.5 \%$ ropivacaine has promoted mean 16 hour analgesia, ${ }^{5}$ slightly lower than our 18 hour result. A comparison of racemic and bupivacaine with levogyrous enantiomeric excess of 50\% (S75: R25) for brachial plexus block, has not shown differences in observed parameters. ${ }^{12}$ Authors concluded that S75: R25 is a safer alternative as compared to racemic bupivacaine due to lower toxicity. ${ }^{12}$

The possibility of epidural and intrathecal injection of anesthetic should be considered. There are several mechanisms that have been invoked to explain events such spinal anesthesia during brachial plexus. Local anesthetics can enter the epidural or spinal space through at least three different routes. First, the drug may be injected directly into the epidural space or intrathecally. Second, a dural cuff may accompany a nerve root some distance distal to the intervertebral foramen through which it passes and then may accidentally be punctured; making direct intrathecal injection possible. ${ }^{13}$ This should be suspected if there is a marked resistance to injection accompanied by pain..$^{13}$ In the present study, there were no cases of subarachnoid or epidural injection with the posterior brachial plexus block technique.

\section{Conclusion}

In conclusion, for clavicle, shoulder and proximal humeral surgeries, posterior brachial plexus block using nerve-stimulation technique and single-injection of local anesthetic proved to be quite comfortable for the patient in a sitting position, easy to perform by marking the spinous process of $\mathrm{C} 6$, being a safe technique and excellent effectiveness. The use of lidocaine infiltration and sedation with $0.1 \mathrm{mg} / \mathrm{kg}$ dextroketamine allowed for pain-free placement 
during A100 needle puncture and no residual neck pain, and analgesia duration of 18 hours.

\section{Funding details}

No financial sources supported this work.

\section{Acknowledgments}

No competing financial interests exist.

\section{Conflicts of interest}

Authors declare that there is no conflict of interest.

\section{References}

1. Brown DL. Brachial plexus anesthesia: an analysis of options. Yale $J$ Biol Med. 1993;66(5):415-431.

2. Kappis M. Conduction anesthesia of abdomen, breast, arm, and neck with paravertebral injection (German). Munchen Med Wochenschr. 1912;59:794-796.

3. Pippa P, Cominelli E, Marinelli C, et al. Brachial plexus block using the posterior approach. Eur J Anaesthesiol. 1990;7:411-420.

4. Boezaart AP, Koorn R, Borene S, et al. Continuous brachial plexus block using the posterior approach. Reg Anesth Pain Med. 2003;28:70-71.

5. Beato L, Camocardi G, Imbelloni LE. Posterior brachial plexus block with nerve stimulator and $0.5 \%$ ropivacaine. Rev Bras Anestesiol. 2005;55:4:421-428.

6. Cruvinel MGC, Castro CHV, Silva YP, et al. A comparative study on the postoperative analgesic efficacy of 20,30, or $40 \mathrm{~mL}$ of ropivacaine in posterior brachial plexus block. Rev Bras Anestesiol. 2007;57(5):500-513.
7. Cruvinel MGC, Castro CHV, Silva YP, et al. Comparative study for the postoperative analgesic efficacy of $20 \mathrm{~mL}$ at $0.5,0.75$, and $1 \%$ ropivacaine in posterior brachial plexus block. Rev Bras Anestesiol. 2008;58(5):431-439.

8. Imbelloni LE, Gouveia MA, de Morais Filho GB. Comparison of the effects of four subdoses of dextroketamine to reduce pain during posterior brachial plexus block: A randomized double blind study. Anesth Essays Res. 2017;11(2):345-349.

9. Boezaart AP. That which we call a rose by any other name would smell as sweet - and its thorns would hurt as much. Reg Anesth Pain Med. 2009;34(1):3-7.

10. Rettig HC, Gielen MJM, Jack NTM, et al. A comparison of the lateral and posterior approach for brachial plexus block. Reg Anesth Pain Med. 2006;31(2):119-126.

11. Sandefo I, Iohom G, Elstraete AV, et al. Clinical efficacy of then brachial plexus block via the posterior approach. Reg Anesth Pain Med. 2005;30(3):238-242.

12. Sato RT, Porsani DF, Amaral AGV, et al. Comparative study of $0.5 \%$ racemic bupivacaine versus enantiomeric mixture (S75-R25) of $0.5 \%$ bupivacaine in brachial plexus block for orthopedic surgery. Rev Bras Anestesiol. 2005;55(2):165-174.

13. Baraka A, Hanna M, Hammoud R. Unconsciousness and apnea complicating parascalene brachial plexus block:possible subarachnoid block. Anesthesiology. 1992;77(5):1046-1047. 